\title{
Introducing State-Trajectory Control for the Synchronous Interleaved Boost Converter
}

\author{
Rafael Peña-Alzola, Peter Ksiazek and Martin Ordonez \\ Department of Electrical and Computer Engineering \\ The University of British Columbia \\ Vancouver, BC V6T 1Z4 Canada \\ E-mails: rafaelpa@ece.ubc.ca \\ peter.ksiazek@ieee.org mordonez@ieee.org
}

\author{
Huai Wang and Frede Blaabjerg \\ Department of Energy Technology \\ Aalborg University \\ DK-9220 Aalborg East, Denmark \\ E-mails: hwa@et.aau.dk \\ fbl@et.aau.dk
}

\begin{abstract}
Synchronous interleaved boost converters (SIBCs) result in lower ripple currents and bidirectional power flow. The boost topology has a non-minimum phase characteristic, producing instability problems when a large bandwidth is required. Linear controllers inherently limit the boost controller bandwidth, resulting in a slow response. In this paper, statetrajectory control of the SIBC based on boundary control is proposed to provide an outstanding dynamic response during start-up and sudden load changes, close to the physical limit of the system. The proposed controller and derivation provides a rigorous framework that deals with four switching states, and three state equations, resulting in a simple control law with very fast dynamic response. The normalized trajectories for the SIBC are determined in the geometric domain along with the control law. The exact trajectories are used for fast transients, and approximate trajectories are employed for constant frequency in steady-state. Simulation and experimental results are provided to validate the proposed procedures.
\end{abstract}

\section{INTRODUCTION}

The basic boost topology has a non-minimum phase characteristic, producing instability problems [1] when a large bandwidth is required. Linear controllers inherently limit the boost controller bandwidth [2], resulting in a slow response as shown in Fig. 1. In order to overcome these issues, nonlinear techniques, such as sliding mode [3]-[5] and boundary control [6]-[9] have been proposed. Boundary control provides an outstanding dynamic response during start-up and sudden load changes [6], [9]. Since the dynamic response is close to the theoretical optimum, steady state can be achieved with very few switching actions [7], [9]. These fast dynamics may enable the use of film capacitors instead of electrolytic capacitors, which would significantly increase the reliability of the system [10]. Boundary control uses the trajectories of the state variables in the phase-plane (geometric) to obtain a control law [11], [12]. The previous literature review [6][8], [11]-[14] indicates that there is an abundance of articles on boundary control for the regular boost converter, but no studies on boundary control of the interleaved boost converter. Boundary control of the SIBC results in increased complexity, with an additional state equation necessitated by the additional storage element, and it is addressed in this work. Additionally, as shown in Fig. 1, the number of switching states is doubled (four switching states of SIBC versus two switching states

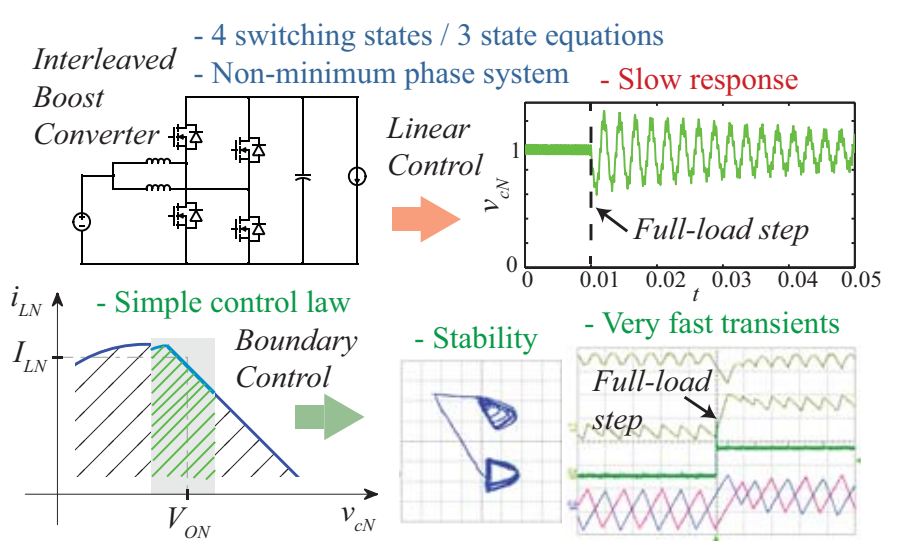

Fig. 1. Conceptual procedure and results of the proposed boundary control for the SIBC.

of simple boost converters). The proposed controller and derivation provides a rigorous framework that deals with the additional states and equations, resulting in a simple control law with very fast dynamic response, which is conceptually depicted in Fig. 1. The normalized trajectories for the SIBC are determined in the geometric domain along with the control law. The exact trajectories are used for fast transients, and approximate trajectories are employed for constant frequency in steady-state. Simulation and experimental results are provided to validate the proposals.

\section{Normalized State TRAJectories}

Fig. 2 shows the bidirectional SIBC; each inductor has an inductance of $2 L$, so the total parallel inductance is $L$. The load is a constant current source $I_{O}$ and the input voltage $V_{I}$ is considered constant (capital letters will denote constant magnitudes). There are four different switching states in the SIBC, with the switches $S_{1}$ and $S_{2}$ being $1 / 0$ when on/off. The lower switches are complementary to the upper ones. The equations of the boost converter depend on the switching state of the power devices $S_{1}$ and $S_{2}$ according to:

$$
C \frac{d v_{c}}{d t}=\bar{S}_{1} i_{L 1}+\bar{S}_{2} i_{L 2}-I_{O}
$$




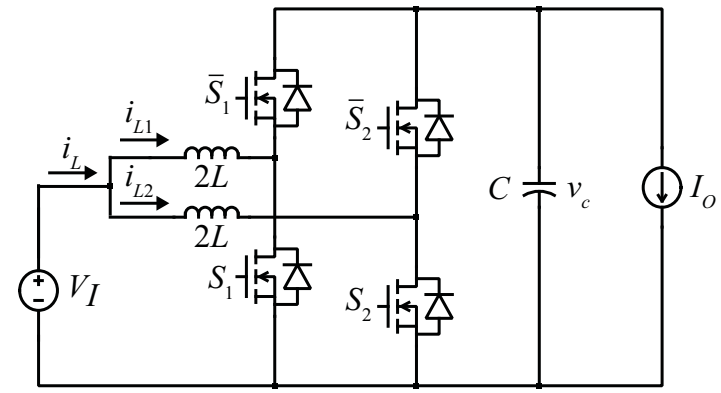

Fig. 2. Synchronous Interleaved boost converter (SIBC).

$$
\begin{aligned}
& 2 L \frac{d i_{L 1}}{d t}=V_{I}-\bar{S}_{1} v_{c} \\
& 2 L \frac{d i_{L 2}}{d t}=V_{I}-\bar{S}_{2} v_{c}
\end{aligned}
$$

with $v_{c}$ the output voltage, $C$ the output capacitor capacitance, and finally $i_{L 1}$ and $i_{L 2}$ the currents through the different inductors. In order to derive a simple control law, it is convenient to define the total current $i_{L}=i_{L 1}+i_{L 2}$ and the current difference $\Delta i_{L}=i_{L 1}-i_{L 2}$. It is also convenient to normalize the voltage, current, and time, with $V_{b}=V_{\text {rated }}$ the output rated voltage, $I_{b}=V_{\text {ref }} \sqrt{C / L}$ and $T_{b}=\sqrt{L C}$ respectively [13]. With this normalization $C_{N}=L_{N}=1$, which allows for simpler formulas and lower computations for the control law implementation. For the switching states $S_{1}=S_{2}$, the following equations result:

$$
\begin{aligned}
& \left.\begin{array}{rl}
\frac{d v_{c N}}{d t_{N}} & =i_{L N}-I_{O N} \\
\frac{d i_{L N}}{d t_{N}} & =V_{I N}-v_{c N} \\
& 2 \frac{d \Delta i_{L N}}{d t_{N}}=0
\end{array}\right\} \text { for } S_{1}=S_{2}=0 \\
& \left.\begin{array}{c}
\frac{d v_{c N}}{d t_{N}}=-I_{O N} \\
\frac{d i_{L N}}{d t_{N}}=V_{I N} \\
2 \frac{d \Delta i_{L N}}{d t_{N}}=0
\end{array}\right\} \text { for } S_{1}=S_{2}=1
\end{aligned}
$$

The subscript $N$ denotes normalized variables. The selected normalization presents another interesting property, namely that the trajectories for $S_{1}=S_{2}=0$ are circles, with their centres at $\left(I_{O N}, V_{I N}\right)$, rotating with unity angular speed, see Fig. 3:

$$
\begin{aligned}
S_{1}=S_{2}= & 0: \\
& \quad\left(i_{L N}-I_{O N}\right)^{2}+\left(v_{c N}-V_{I N}\right)^{2} \\
& =\left(I_{L B N}-I_{O N}\right)^{2}+\left(V_{c B N}-V_{I N}\right)^{2}
\end{aligned}
$$

with $I_{L N B}, \Delta I_{L N B}$ and $V_{c B N}$ the initial values. The trajectories for $S_{1}=S_{2}=1$ are straight lines, see Fig. 3, [13]:

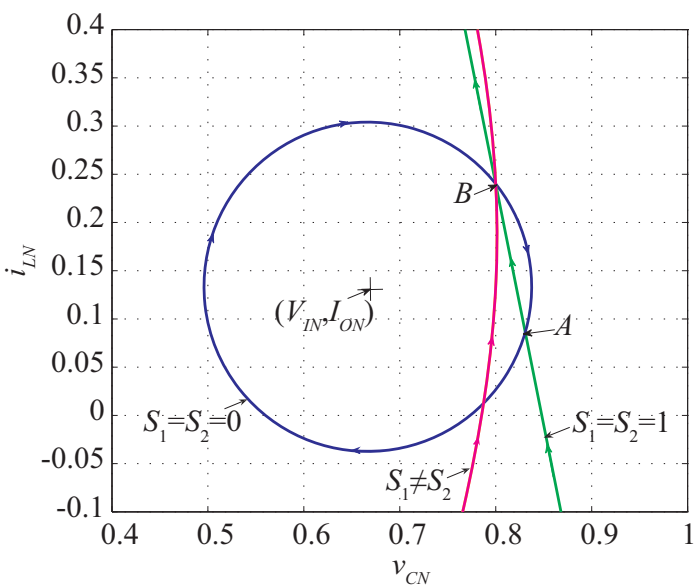

Fig. 3. Trajectories for the different switching states.

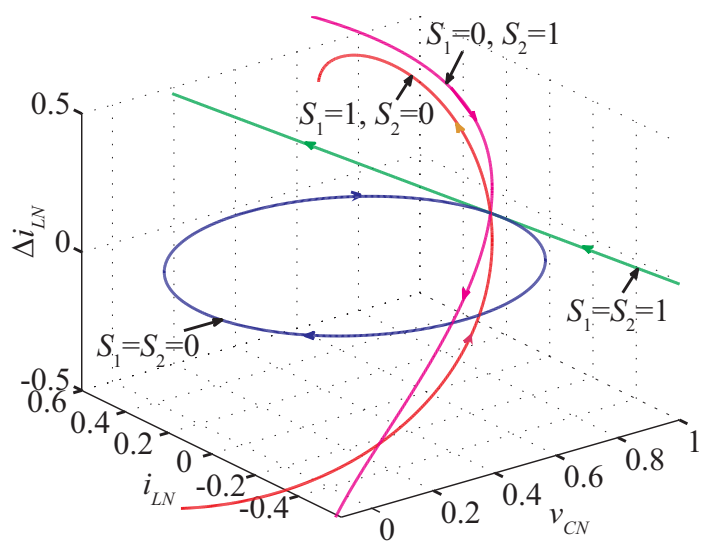

Fig. 4. Trajectories for the different switching states including $\Delta i_{L N}$.

$$
\begin{aligned}
& S_{1}=S_{2}=1: \\
& \quad\left(i_{L N}-I_{L N B}\right)=-V_{I N} / I_{O N}\left(V_{c N}-V_{c B N}\right)^{2}
\end{aligned}
$$

It is clear that, for these cases, the SIBC behaves as a simple boost converter. Moreover, the current difference $\Delta i_{L}$ does not change for this switching states $S_{1}=S_{2}$, see (2)-(3). For $S_{1} \neq S_{2}$, the following equations result:

$$
\begin{aligned}
& \left.\frac{d v_{c N}}{d t_{N}}=\frac{1}{2}\left(i_{L N}-\Delta i_{L N}\right)-I_{O N}\right) \\
& \left.\begin{array}{c}
\frac{d i_{L N}}{d t_{N}}=V_{I N}-\frac{v_{O N}}{2} \\
2 \frac{d \Delta i_{L N}}{d t}=v_{c N}
\end{array}\right\} \text { for } S_{1}=1, S_{2}=0 \\
& \left.\frac{d v_{c N}}{d t_{N}}=\frac{1}{2}\left(i_{L N}+\Delta i_{L N}\right)-I_{O N}\right) \\
& \left.\frac{d i_{L N}}{d t_{N}}=V_{I N}-\frac{v_{c N}}{2}\right\} \text { for } S_{1}=0, S_{2}=1 \\
& 2 \frac{d \Delta i_{L N}}{d t}=-v_{c N}
\end{aligned}
$$


The second equation in (6) and (7) shows that the behavior of $i_{L N}$, increasing or decreasing, depends on whether $V_{I N}$ is higher or lower than $v_{c N} / 2$. The third equation in (6) and (7) shows that $\Delta i_{L N}$ varies when these switching states $S_{1} \neq S_{2}$ are applied. By eliminating $i_{L N}$ and $\Delta i_{L N}$ in the first equation of the previous sets, it results that:

$$
\left.\begin{array}{r}
2 \frac{d^{2} v_{c N}}{d t_{N}^{2}}+v_{c N}=V_{I N} \\
\left.\begin{array}{r}
\frac{d i_{L N}}{d t_{N}}=V_{I N}-\frac{v_{c N}}{2} \\
2 \frac{d \Delta i_{L N}}{d t}=v_{c N}
\end{array}\right\} \text { for } S_{1}=1, S_{2}=0 \\
2 \frac{d^{2} v_{c N}}{d t_{N}^{2}}+v_{c N}=V_{I N} \\
\frac{d i_{L N}}{d t_{N}}=V_{I N}-\frac{v_{c N}}{2} \\
2 \frac{d \Delta i_{L N}}{d t}=-v_{c N}
\end{array}\right\} \text { for } S_{1}=0, S_{2}=1
$$

Therefore, according to (8) and (9), the switching states $S_{1} \neq S_{2}$ produce the same effect for $i_{L N}$, but the opposite for $\Delta i_{L N}$. Moreover, the trajectories in the phase plane of $v_{c N}$ and $i_{L N}$ for $S_{1} \neq S_{2}$ will be the same, see Fig. 3, provided that the initial values are the same. In the normalized domain, the phase plane trajectories of these equations result in a circle rotating at a constant speed for $i_{L N 1}$ and a straight line for $i_{L N 2}$. The values for $i_{L N}$ and $\Delta i_{L N}$ result from the combination of $i_{L 1}$ and $i_{L 2}$, but the trajectory equations do not result in simple geometrical forms, see Fig. 3. The three dimensional trajectories for the different switching states are shown in Fig. 4. It can be seen that, unlike the trajectories for $S_{1} \neq S_{2}$, the trajectories for states $S_{1}=S_{2}$ are in a plane, as $\Delta i_{L N}$ does not vary.

\section{Steady State Behavior}

For transients, the switching states $S_{1}=S_{2}$ should be used and the interleaved converter will behave like a standard boost. At steady state, the SIBC alternates the switching states $S_{1} \neq S_{2}$ to minimize the current ripple, and this is the same as selecting the most propitious switching sequence. In order to make the previous equations analytically tractable at steady state, it is considered that $v_{c N} \approx V_{O N}$ [11] with $V_{O N}$ the average value of the output voltage. This is the same as assuming a proper voltage regulation [11] with the currents being straight lines. With $v_{c N} \approx V_{O N}$, the trajectory for $S_{1}=S_{2}=0$ is a parabola approximating the circle (4) according to [12]:

$$
\begin{aligned}
& S_{1}=S_{2}=0: \\
& \quad v_{c N}=v_{B N}-\frac{i_{L N}^{2}-i_{B N}^{2}}{2\left(V_{O N}-V_{I N}\right)}+I_{O N} \frac{i_{L N}-i_{B N}}{V_{O N}-V_{I N}}
\end{aligned}
$$

For $S_{1}=1, S_{2}=0$, the trajectory is also a parabola by approximating the complex curve resulting from solving (8)(9):

$$
\begin{aligned}
& S_{1}=1, S_{2}=0: v_{C N}=v_{C N B} \\
& +\frac{\left(i_{L N}-i_{L N B}\right) V_{O N}\left(\Delta i_{L N B}-i_{L N}+2 I_{O N}\right)}{\left(2 V_{I N}-V_{O N}\right)^{2}} \\
& -\frac{V_{I N}\left(i_{L N}-i_{L N B}\right)\left(2 \Delta i_{L N B}-i_{L N}-i_{L N B}+4 I_{O N}\right)}{\left(2 V_{I N}-V_{O N}\right)^{2}}
\end{aligned}
$$

The solution for $S_{1}=0, S_{2}=1$ is the same as the solution for (11) but with the opposite sign for $\Delta i_{L N B}$. Moreover, $\Delta i_{L N}$ increases linearly with slope $V_{O N} / 2$ for $S_{1}=1, S_{2}=0$ and decreases for $S_{1}=0, S_{2}=1$,

$$
\Delta i_{L N}=\Delta i_{L N}(0) \pm \frac{V_{O N} t_{N}}{2} \text { for } S_{1}=1 / 0, S_{2}=0 / 1
$$

For $V_{I N}<V_{O N} / 2$, the sequences $S_{1} \neq S_{2}$ result in decreased $i_{L N}$, see Fig. 5, just as occurs with $S_{1}=S_{2}=0$. Hence, the switching states used in this case are $S_{1} \neq S_{2}$ to decrease $i_{L N}$ and $S_{1}=S_{2}=1$ to increase it. The state $S_{1}=S_{2}=0$ will not be used during the steady state. The points $B$ and $A$ of maximum and minimum current correspond to the final and initial application of $S_{1}=S_{2}=1$ respectively, see Fig. 5. The trajectories for $V_{I N}>V_{O N} / 2$ are too close to be discerned in a practical implementation, and will not be considered.

To calculate the relation between point $B\left(I_{L N B}, V_{c N B}\right)$ in the phase plane with minimum current and the average point $\left(I_{L N}, V_{O N}\right)$, see Fig. 5, three conditions must be met, namely, the power must be conserved, and the average current and voltage must be calculated [15]:

$$
V_{I N} I_{L N}=V_{O N} I_{O N}
$$

$$
\begin{aligned}
& I_{L N}=\frac{1}{T_{s w}}(\underbrace{\int_{0}^{T_{B \rightarrow A}} i_{L N} d t}_{B \rightarrow A\left(S_{1}=1, S_{2}=0\right)}+\underbrace{\int_{0}^{T_{A \rightarrow B}} i_{L N} d t}_{A \rightarrow B\left(S_{1}=1, S_{2}=1\right)}) \\
& V_{O N}=\frac{1}{T_{s w}}(\underbrace{\int_{0}^{T_{B \rightarrow A}} v_{c N} d t}_{B \rightarrow A\left(S_{1}=1, S_{2}=0\right)}+\underbrace{\int_{0}^{T_{A \rightarrow B}} v_{c N} d t}_{A \rightarrow B\left(S_{1}=1, S_{2}=1\right)})
\end{aligned}
$$

with $T_{B \rightarrow A}$ the time corresponding to descending current, see Fig. 5, from $B$ to $A$. Finally, $T_{s w N}$ is the switching period. Therefore, for $B \rightarrow A$, the solutions for the equations $S_{1} \neq S_{2}$ (6)-(7) must be used and for $A \rightarrow B$, the solution for equation $S_{1}=S_{2}=1$ equation (3) must be used. Additionally, it is necessary to use the condition of periodicity $i_{L N}\left(T_{s w N}\right)=$ $i_{L N B}$ [15]. Finally, in steady state the average value of $\Delta i_{L N B}$ must be null. Because the switching states $S_{1} \neq S_{2}=1$ are applied alternately, the following constraint must be fulfilled: 


$$
0=\frac{1}{2 T_{s w}}(\underbrace{\int_{0}^{T_{B \rightarrow A}} \Delta i_{L N} d t}_{B \rightarrow A\left(S_{1}=1, S_{2}=0\right)}+\underbrace{\int_{0}^{T_{B \rightarrow A}} \Delta i_{L N} d t}_{B \rightarrow A\left(S_{1}=0, S_{2}=1\right)})
$$

After some algebraic operations, the relation between the average values and the values at the extreme point $B$ is:

$$
\begin{gathered}
V_{c N B}=V_{O N}+\frac{I_{O N} T_{s w N}\left(2 V_{I N}-V_{O N}\right)}{2 V_{O N}} \\
+\frac{T_{s w N}{ }^{2} V_{I N}{ }^{3}\left(V_{I N}-V_{O N}\right)}{3 V_{O N}{ }^{3}} \\
I_{L N B}=\frac{I_{O N} V_{O N}}{V_{I N}}-\frac{T_{s w N} V_{I N}\left(2 V_{I N}-V_{O N}\right)}{2 V_{O N}}
\end{gathered}
$$

The maximum $\Delta i_{L N B}$ corresponding to the points $B$ and $A$ is:

$$
\Delta i_{L N \max }= \pm \frac{T_{s w N} V_{I N}}{2}
$$

\section{Control Law Derivation}

Boundary control uses the solution of the previous equations to establish an appropriate control law to achieve an outstanding dynamic response. For large transients, with $v_{C N}$ far from the reference $V_{O N}$, the boost converter should react as quickly as possible. This is achieved by using the switching states $S_{1}=S_{2}$ as a simple boost converter. The switching surface is determined by the circle (4) and the straight line (5) intersecting at the point $B$, the coordinates of which are calculated in (17)-(18), see Fig. 6. Therefore, the first control law is:

$$
\begin{aligned}
v_{c N}<v_{c N L} \text { or } v_{c N}>v_{c N H}: & \\
\text { if }\left(v_{c N}, i_{L N}\right) \in L \text { then } S_{1}=S_{2} & =1 \\
\text { else } S_{1} & =S_{2}=0
\end{aligned}
$$

Note again that, during these switching actions, $\Delta i_{L N}$ remains constant. Finally, when the trajectory is near the ripple area in the state plane, see the shaded area $s$ in Fig. 6, the switching state $S_{1}=S_{2}=0$ is replaced by the sequence $S_{1} \neq S_{2}$ alternating after $S_{1}=S_{2}=1$ to make the average $\Delta i_{L N}$ null:

$$
\begin{aligned}
& v_{c N}>v_{c N L} \text { and } v_{c N}<v_{c N H}: \\
& \text { if }\left(i_{L N}, v_{c N}\right) \in S \text { then } S_{1}=S_{2}=1 \\
& \quad \text { else alternate } S_{1}=0 / 1, S_{2}=1 / 0
\end{aligned}
$$

In order to prevent chatter, a hysteresis band is needed for $v_{C N L}$ and $v_{c N H}$. It can be seen that, despite the additional state in the equations of the SIBC, the control law is simple to implement.

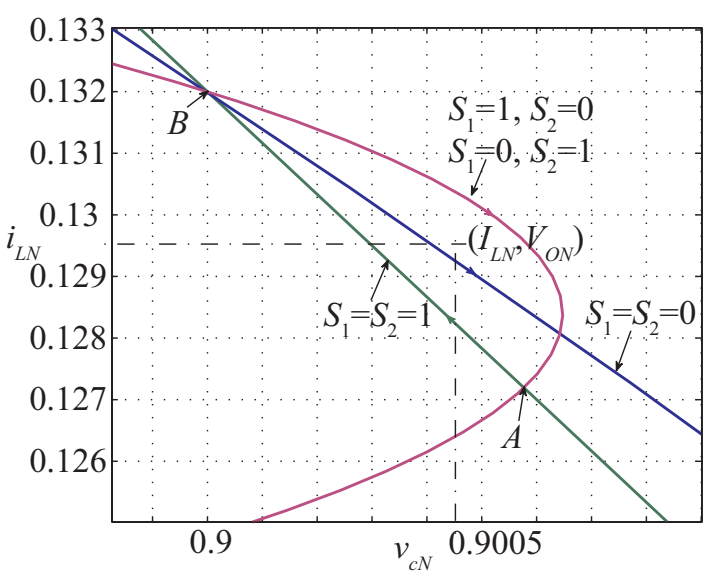

Fig. 5. Trajectories near the operating point for $V_{I N}<v_{c N} / 2$.

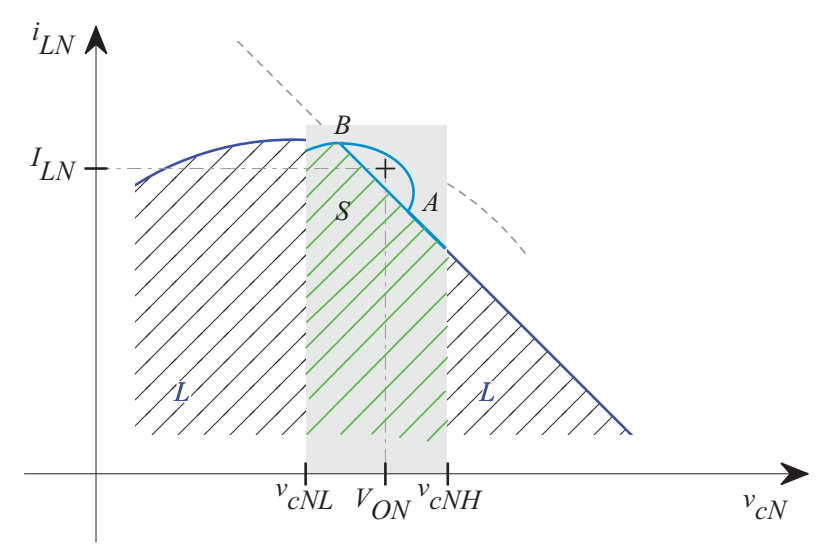

Fig. 6. Representation of the control law.

\section{Simulation And Experimental Results}

Table I contains the parameters for the simulations and the experiments, based on a converter operating at moderate switching frequency. Figs. 7 and 8 show the simulation results for a full-load step, achieving very fast transient even in the presence of low switching frequency. The switching devices are modeled ideally. The ESR of the passive elements, and the quantization effects of the ADCs, are considered. Fig. 7 shows the signals in the time domain, and Fig. 8 shows the phase plane for $i_{L N}$ and $v_{c N}$. The actions of the boundary control allow for a very fast transient with very low dip in the output voltage even when using small film capacitors. It can also be seen that, after the transient, the interleaving switching sequence resumes with very close to constant switching frequency during steady state.

Figs. 9 and 10 show the experimental results for the same previous full-load step. All the calculations of the simple control law are performed using a low cost DSP (TMS320F28335 by Texas Instruments), which was programmed using C. Fig. 9 shows the signals in the time domain, and Fig. 10 the phase plane for $v_{C N}$ and $i_{L N}$. It can be seen that the experimental results are very similar to the previous simulation results, and 
TABLE I

PARAMETERS FOR THE SIMULATIONS AND EXPERIMENTS

\begin{tabular}{lc}
\hline Parameter & Value \\
\hline Rated power $P_{n}$ & $60 \mathrm{~W}$ \\
Output voltage $V_{O}$ & $25 \mathrm{~V}$ \\
Input voltage $V_{I}$ & $10 \mathrm{~V}$ \\
Switching frequency $f_{s w}$ & $3 \mathrm{kHz}$ \\
Sampling frequency $f_{s}$ & $300 \mathrm{kHz}$ \\
Inductor inductance $L$ & $0.508 \mathrm{mH}$ \\
Inductor resistance $R_{L}$ & $36 \mathrm{~m} \Omega$ \\
Capacitor capacitance $C$ & $141 \mu \mathrm{F}$ \\
Capacitor resistance $R_{C}$ & $9.7 \mathrm{~m} \Omega$ \\
\hline
\end{tabular}

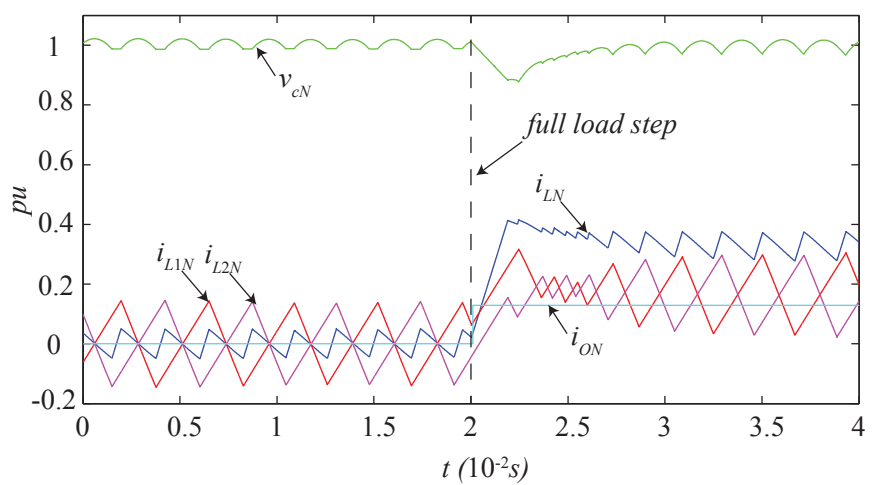

Fig. 7. Simulation results for the full load step: time domains signals $v_{C N}$, $i_{L N}, i_{L 1 N}, i_{L 2 N}$ and load current $i_{O N}$.

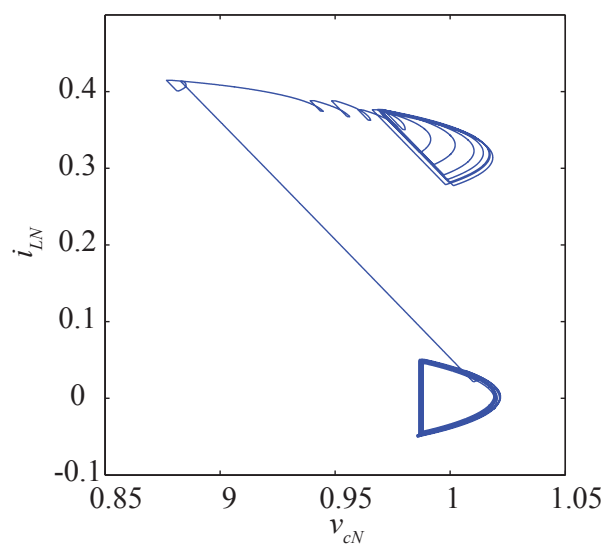

Fig. 8. Simulation results for the full load step: phase plane for $v_{C N}$ and $i_{L N}$.

show a very fast response. The bandwidth limitation of the sensors and the full losses of the passive elements have a smoothing effect on the control magnitudes. This leads to fewer switching actions after the load step comparing to the simulations. It also leads to small variations in the switching actions during steady state with approximately constant switching frequency.

\section{CONCLUSION}

This paper has introduced the boundary control of the synchronous interleaved boost inverter. This control strategy

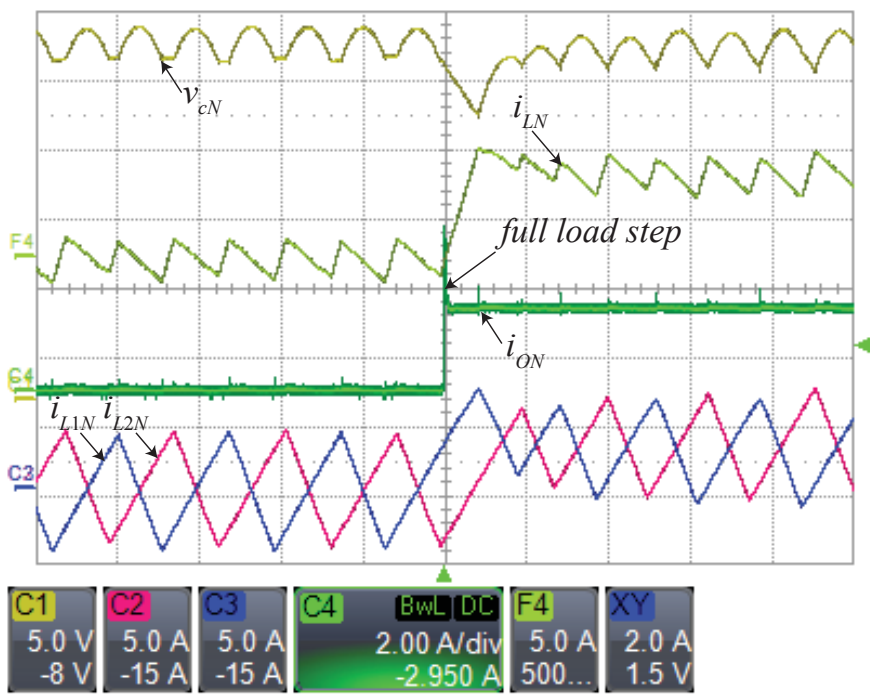

Fig. 9. Experimental results for the full load step: time domains signals $v_{C N}$, $i_{L N}, i_{L 1 N}, i_{L 2 N}$.
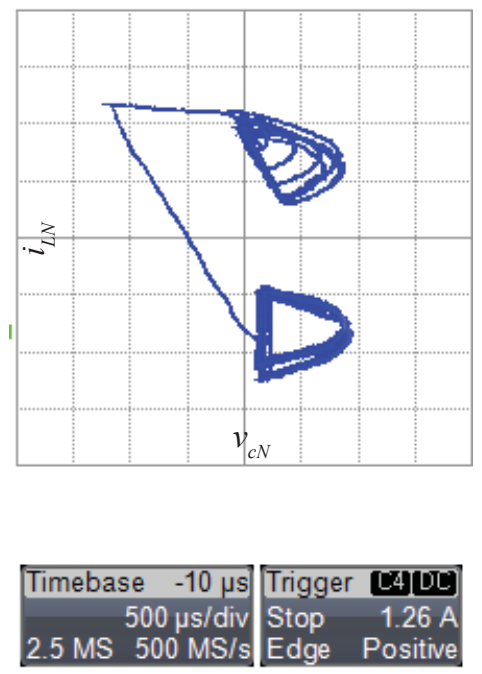

Fig. 10. Experimental results for the full load step: phase plane for $v_{C N}$ and $i_{L N}$.

enables the non-minimum phase problems of the boost converter to be overcome, and it is able to cope with the presence of additional switches and passive elements in the interleaved converter. The derived control law is simple and is based on the rigorous analysis of the exact trajectories during transients, and approximate trajectories during steady state. The simulations and experiments, which are fully consistent with the theoretical derivations, validate the proposals and result in very fast transients for full-load step variations.

\section{REFERENCES}

[1] R. W. Erickson and D. Maksimovic, Fundamentals of Power Electron. Springer, 2001.

[2] Z. Chen, W. Gao, J. Hu, and X. Ye, "Closed-loop analysis and cascade control of a nonminimum phase boost converter," IEEE Trans. Power Electron., vol. 26, no. 4, pp. 1237-1252, April 2011. 
[3] L. Martinez-Salamero, G. Garcia, M. Orellana, C. Lahore, and B. Estibals, "Start-up control and voltage regulation in a boost converter under sliding-mode operation," IEEE Trans. Ind. Electron., vol. 60, no. 10, pp. 4637-4649, Oct 2013 .

[4] S. Oucheriah and L. Guo, "Pwm-based adaptive sliding-mode control for boost dc-dc converters," IEEE Trans. Ind. Electron., vol. 60, no. 8 , pp. 3291-3294, Aug 2013.

[5] R. Giral, L. Martinez-Salamero, R. Leyva, and J. Maixe, "Slidingmode control of interleaved boost converters," IEEE Trans. Circuits and Systems I: Fundamental Theory and Applicat., vol. 47, no. 9, pp. 13301339, Sep 2000

[6] R. Munzert and P. T. Krein, "Issues in boundary control [of power convertors]," in IEEE PESC '96, vol. 1, Jun 1996, pp. 810-816 vol.1.

[7] M. Greuel, R. Muyshondt, and P. T. Krein, "Design approaches to boundary controllers," in IEEE PESC '97, vol. 1, Jun 1997, pp. 672-678 vol.1.

[8] G. E. Pitel and P. T. Krein, "Trajectory paths for dc - dc converters and limits to performance," in IEEE COMPEL '06, July 2006, pp. 40-47.

[9] P. T. Krein, Elements Of Power Electronics. Oxford University Press, Incorporated, 1998

[10] G. M. Buiatti, J. A. Martin-Ramos, C. H. R. Garcia, A. Amaral, and A. J. Marques Cardoso, "An online and noninvasive technique for the condition monitoring of capacitors in boost converters," IEEE Trans. Instrumentation and Measurement, vol. 59, no. 8, pp. 2134-2143, Aug 2010.

[11] W. W. Burns and T. G. Wilson, "State trajectories used to observe and control dc-to-dc converters," IEEE Trans. Aerosp. Electron. Syst., vol. AES-12, no. 6, pp. 706-717, Nov 1976.

[12] — "A state-trajectory control law for dc-to-dc converters," IEEE Trans. Aerosp. Electron. Syst., vol. AES-14, no. 1, pp. 2-20, Jan 1978.

[13] J. M. Galvez, M. Ordonez, F. Luchino, and J. E. Quaicoe, "Improvements in boundary control of boost converters using the natural switching surface," IEEE Trans. Power Electron., vol. 26, no. 11, pp. 3367-3376, Nov 2011.

[14] T.-T. Song and H. S. H. Chung, "Boundary control of boost converters using state-energy plane," IEEE Trans. Power Electron., vol. 23, no. 2, pp. 551-563, March 2008.

[15] W. W. Burns, "A theory of control for a class of electronic power processing systems," Ph.D. dissertation, Duke Univ., Durham, NC., April 1977. 